\title{
ROCKING MOTION OF SLENDER ELASTIC BODY ON RIGID FLOOR
}

\author{
T. Ichinose*
}

\begin{abstract}
A single degree of freedom (SDOF) model is presented to describe the rocking behaviour of a slender elastic body on a rigid floor. This model assumes the vibration mode to be a linear combination of flexural and rocking modes. A finite element analysis is also presented, in which following features are observed to support the assumptions of the proposed SDOF model: (1) There exists a simple relationship between the magnitudes of flexural and rocking modes, which relation can be derived from the equilibrium of moment. (2) Angular momentum is conserved at the instances of uplifting and landing.
\end{abstract}

\section{INTRODUCTION}

Seismic hazards within buildings can be caused not only by structural damages but also by overturning or collapse of containing articles. During the 1978 Miyagi-ken-oki earthquake in Japan, a lot of stacked crates containing bottles of beer or soft drinks collapsed in warehouses and factories, causing economic losses and a menace to the safety of workers there (i).

A number of experimental and analytical studies have contributed to an understanding of the rocking motion of rigid bodies $(2,4,6)$. However, studies on the dynamic properties of the rocking motion of elastic bodies are few. In the case of the 1978 Miyagi-ken-oki earthquake cited above, the collapsed stacks of crates were so flexible that the fundamental periods prior to rocking were longer than $1.0 \mathrm{sec}(5)$. It is thus clear that modelling them as rigid bodies would be inappropriate, and that a methodology should be developed to study the response of elastic rocking bodies.

The objective of this paper is to develop a single degree of freedom (SDOF) model to describe the rocking behaviour of a slender elastic body on a rigid floor. Results of finite element analysis are also presented to verify the assumptions of the SDOF modelling.

2. SINGLE DEGREE OF FREEDOM (SDOF) MODEL

2.1 Analytical Assumptions

\subsubsection{Assumption 1}

Plane sections of the body remain plane (Navier's hypothesis), and the total work done by following forces during virtual horizontal displacement $\delta u$ is zero (principle of virtual work).

(1) Inertia force

*Visitor, Department of Civil Engineering University of Canterbury, Christchurch New zealand (on leave from Department of Architecture Nagoya Institute of Technology, Nagoya, Japan)
(2) Second derivative of bending moment due to elasticity and strain rate damping.

In other words, the following equation is assumed.

$$
\begin{aligned}
\int_{0}^{H}\left[\rho \cdot\left(\frac{\partial^{2} u}{\partial t^{2}}+\frac{d^{2} u}{d t^{2}}\right)+\gamma \cdot I \cdot \frac{\partial^{5} u}{\partial t \partial y^{4}}\right. \\
\left.+E I \cdot \frac{\partial^{4} u}{\partial y^{4}}\right] \cdot \delta u \cdot d y=0
\end{aligned}
$$

$$
\text { where } \begin{aligned}
\rho=M / H= & \text { mass per unit height } \\
M \text { and } H= & \begin{array}{l}
\text { total mass and height of the } \\
\text { body }
\end{array} \\
u=u(y, t)= & \begin{array}{l}
\text { horizontal displacement } \\
\text { relative to the floor }
\end{array} \\
y \text { and } t= & \text { vertical coordinate and time } \\
u_{0}=\nu_{0}(t)= & \text { displacement of the floor } \\
\gamma & =\begin{array}{l}
\text { damping coefficient per unit } \\
\text { strain rate }
\end{array} \\
I & \text { moment of inertia of section } \\
E & =\text { Young's modulus }
\end{aligned}
$$

Note that the expression in the squarebracket [] equated to zero gives an equation of motion for a flexural beam with distributed mass (3).

Navier's hypothesis requires the rocking motion to occur around the toe of the elastic body as shown in Fig.1(a). Therefore, the axis of centroid of the body is lifted up during rocking. However, effects of vertical movement are ignored in Eq. (1) for simplicity. This disregard is justified only when the body is slender enough that $(B / H)^{2}=0$ (where $B$ and $H$ are width and height of the body) as will be shown within the appendix about the relationship between the presented SDOF model and the classical theory for rigid bodies. 


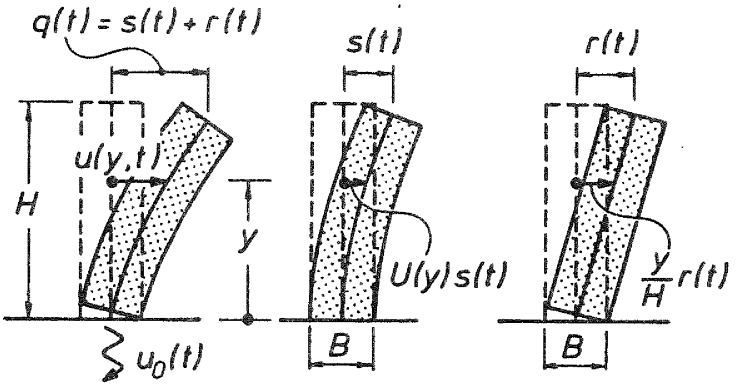

\section{(a) Total Displacement \\ (c) Rocking Component}

\section{Figure 1: Assumed vibration mode}

\subsubsection{Assumption 2}

Horizontal displacement of the body relative to the floor, $u$, can be expressed by a linear combination of the pre-rocking fundamental mode of the uniform cantilever beam $U(y)$, and the rocking mode $(y / H)$, as shown in Fig.1.

$$
u(y, t)=U(y) \cdot s(t)+(y / H) \cdot r(t)
$$

where $s(t)$ and $r(t)$ are flexural and rocking components of the top displacement $q(t)$, which satisfies the following equation.

$$
q(t)=s(t)+r(t)
$$

The cantilever beam mode shape $U(y)$ can be expressed in the following form as shown in Re?.(3).

$$
\begin{aligned}
& \begin{aligned}
U(y)=L_{s} & (\cosh \lambda \xi-\cos \lambda \xi) \\
& \quad-L_{c}(\sinh \lambda \xi-\sin \lambda \xi)
\end{aligned} \\
& \text { where } \quad \begin{aligned}
& \xi=y / H \quad \lambda=1.8751 \\
& L_{s}=1 / 2 \quad, \quad L_{c}=0.3671
\end{aligned}
\end{aligned}
$$

\subsubsection{Assumption 3}

Uplifting occurs when the equilibrium is realised between the elastic bending moment $E I\left(\partial^{2} u / \partial y^{2}\right)$ at the base and the resisting moment due to the gravity force around the rotating axis, which is the toe of the cantilever. The equilibrium remains valid as long as the rocking motion exists $(r(t) \neq 0)$.

\subsubsection{Assumption 4 (Exception to Assumption 1)}

At the instance of landing, the angular momentum is conserved around the edge of landing (point $P$ in Fig.2(a)). At the instance of uplifting, the angular momentum is conserved around the axis of rotation (point $P$ in Fig.2(b)). Therefore, the velocity of the body changes at the instances of landing and uplifting discontinuously, both resulting in decrease of kinetic energy. Effects of vertical movement should also be considered to calculate the angular momentum.

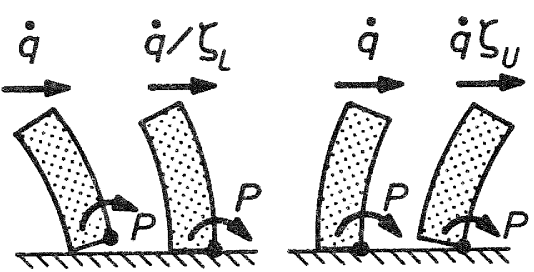

(a) Landing

\section{Figure 2: Conservation of angular} momentum

\subsection{Flexural and Rocking Components of Top} Displacement

From Assumptions 2 and 3 (on mode shape and moment equilibrium), the flexural and rocking components, $s(t)$ and $r(t)$, during rocking motion, can be expressed in terms of the top displacement $q(t)$.

The resisting moment $m_{1}$ by the gravity force around the toe of the body is expressed as follows.

$$
\begin{aligned}
m_{R} & = \pm \frac{1}{2} M \cdot g \cdot B-\rho \cdot g \cdot \int_{0}^{H} u \cdot d y \\
& =\frac{M \cdot B}{2}\left[ \pm B-\frac{4 L c}{\lambda} \cdot s(t)-r(t)\right]
\end{aligned}
$$

where $B$ is the width of the body and has positive sign for $q(t)>0$.

The elastic bending moment at the basement $m_{B}$ is equivalent to the overturning moment $m_{T}$ due to the inertia plus damping force $w$. Now

$$
\begin{aligned}
w & =-\rho\left[\frac{\partial^{2} u}{\partial t^{2}}+\frac{d^{2} u_{0}}{d t^{2}}\right]-\gamma \cdot I \cdot \frac{\partial^{5} u}{\partial t \partial y^{4}} \\
& =E I \cdot \frac{\partial^{4} u}{\partial y^{4}}=E I \cdot\left(\frac{\lambda}{H}\right)^{4} \cdot U(y) \cdot s(t)
\end{aligned}
$$

Hence

$$
m_{T}=\int_{0}^{H} w \cdot y \cdot d y=E I \cdot\left(\frac{\lambda}{H}\right)^{2} \cdot s(t)=m_{B}
$$

where partial integration technique was utilised.

The resisting moment $\mathrm{m}_{\mathrm{R}}$ is always larger than $m_{B}$ as long as $|q|<s_{n}$, where

$$
s_{n}=\frac{B / 4}{\frac{E I}{2 M g} \cdot\left(\frac{\lambda}{H}\right)^{2}+\frac{L_{c}}{\lambda}}
$$

$s_{n}=$ top deflection at the instance of uplift.

Therefore, the rocking motion does not occur while $|q|<s_{n}$, i.e. 


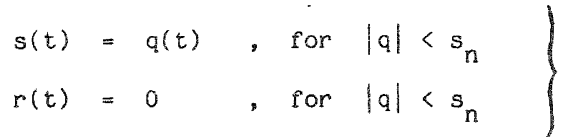

During rocking (i.e., $|q|>s_{n}$ ), the equilibrium of moment requires $m_{B}=m_{B}$ Equating (5) and (7), noting that $s(t)+r(Q)=q(t)$. substituting for $s$ and separating the variables yields the following equations to prescribe $s(t)$ and $r(t)$ during rocking.

$$
\left.\begin{array}{ll}
s(t)=\frac{ \pm B-q(t)}{B-s_{n}} \cdot s_{n}, & \text { for }|q|>s_{n} \\
r(t)=\frac{q(t) \mp s_{n}}{B-s_{n}} \cdot B & \text {, for }|q|>s_{n}
\end{array}\right\}
$$

[The alternative signs depend on the sign of $q(t)$.

\subsection{Reduced SDOF Equation of Motion}

The reduced SDOF equation of motion is obtained from the assumptions 1 and 2 (on energy principle and mode shape) and the results of the previous section.

Substituting the mode shape assumption Eq. (2) into the virtual work principle Eq.(1) and utilising Eqs. (9a) and (9b) gives the following equation.

$\rho \cdot \ddot{q}+\gamma \cdot v \cdot I \cdot\left(\frac{\lambda}{H}\right)^{4} \cdot \dot{s}+v \cdot E I \cdot\left(\frac{\lambda}{H}\right)^{4} \cdot s$

$$
=-\beta \cdot \rho_{0} \ddot{u}
$$

where $\nu$ and $\beta$ are called as effectiveness factor and participation factor, respectively, and defined by the following equations, i.e. before uplifting $\left(|q|<s_{n}\right)$

$\left.\begin{array}{rl}v=v_{1} & =1.0 \\ \text { and } B=\beta_{q} & =\int_{0}^{H} U \cdot d y / \int_{0}^{H} U^{2} \cdot d y=1.564,\end{array}\right\}$

while, during uplifting $\left(|q|>s_{n}\right)$

$$
\left.\begin{array}{rl}
v & =v_{2}=\int_{0}^{H} U \cdot V \cdot d y / \int_{0}^{H} v^{2} \cdot d y<v_{1} \\
\text { and } \beta & =\beta_{2}=\int_{0}^{H} v \cdot d y / \int_{0}^{H} v^{2} \cdot d y<\beta_{1}
\end{array}\right\}
$$

where

$$
V=V(y)=-\frac{s_{n}}{B-s_{n}} U(y)+\frac{B}{B-s_{n}} \cdot \frac{y}{H}
$$

is the velocity distribution mode during uplifting. The integrals in Eqs. (13) have the following values:

$$
\left.\begin{array}{l}
\int_{0}^{H} U \cdot v \cdot d y=\frac{H}{B-s_{n}} \cdot\left(-0.2503 \cdot s_{n}+B / \lambda^{2}\right) \\
\int_{0}^{H} V \cdot d y=\frac{H}{B-s_{n}} \cdot\left(-0.3915 \cdot s_{n}+B / 3\right) \\
\int_{0}^{H} v^{2} \cdot d y=\frac{H}{\left(B-s_{n}\right)^{2}} \cdot\left(0.2503 \cdot s_{n}^{2}-2 \cdot B \cdot s_{n} / \lambda^{2}\right.
\end{array}\right\}
$$

The approximate values of $v_{2}$ and $\beta_{2}$ for $\left(s_{n} / B\right)=0$ to 0.2 are $v_{2} \approx 0.85$ to 0.65 and $B_{2}{ }^{n}=1.5$ to 1.2 . Multiplying both sides of Eq. (10) by $\int_{0}^{H} U . d y=$
$0.3915 . H$, gives

$M_{e} \cdot \ddot{q}+\frac{\gamma}{E} \cdot v \cdot K_{e} \cdot \dot{s}+v \cdot K_{e} s=-B \cdot M_{e} \cdot \ddot{u}_{0}$

where

$M_{e}=\rho \cdot \int_{0}^{H} U \cdot d y=0.3915 \cdot M$

$K_{e}=E I \cdot\left(\frac{\lambda}{H}\right)^{4} \cdot \int_{0}^{H} U \cdot d y=0.4840 \cdot \frac{E I}{H^{4}}$

Expressing $s$ in terms of $q$, we obtain the final shape of the reduced SDOF equation of motion.

$M_{e} \cdot \ddot{q}+\frac{\lambda}{E} \cdot \nu \cdot \frac{d F(q)}{d q} \cdot \dot{q}+v \cdot F(q)=-B \cdot M_{e} \cdot \ddot{u}_{0}$

where $F(q)$ is a nonlinear elastic function given in Fig.3(a): before uplifting $\left(|q|<s_{n}\right)$

$$
F(q)=K_{e} \cdot q
$$

while, during uplifting,

$$
F(q)=k_{e}: \frac{ \pm B-q}{B-s_{n}}: s_{n}
$$

It should be noted that $F(q)$ is the base shear rorce, since

$$
F(q)=E I \cdot \frac{\partial^{3} u}{\partial y^{3}} \quad y=0
$$

The effective base shear force $v . F(q)$ is also shown in Fig.3(b).

If the Young modulis $E$ becomes infinitely large, the derived SDOF model, Eq. (16), converges to the classical equation of motion for slender rigid bodies as shown in the Appendix.

\subsection{Conservation of Angular Momentum}

At the instance of uplifting shown in Fig.2(b), the top velocity decreases from $\dot{q}$ to $\zeta_{\mathrm{U}} / q$. due to the conservation of angular momentum (Assumption 4). The angular momentiom around the axis of rotation $P$ just before and after uplift is denoted as $G_{B U}$ and $G_{A U}$ respectively. They are calculated as follows:

$G_{B U}=p \cdot q \cdot\left[\int_{0}^{H} U \cdot y \cdot d y+\frac{B^{2}}{12}\right]$

$\left.G_{A U}=\rho \cdot \zeta_{U} \cdot \dot{q} \cdot\left[\int_{0}^{H} v \cdot y \cdot d y+\frac{B^{2}}{12}+\frac{B^{2}}{4} \cdot \frac{B}{B-s_{n}}\right]\right\}$

where

$$
\left.\begin{array}{l}
\int_{0}^{H} U \cdot y \cdot d y=\left(\frac{H}{\lambda}\right)^{2} \\
\text { and } \\
\int_{0}^{H} V \cdot y \cdot d y=-\frac{s_{n}}{B-s_{n}} \cdot\left(\frac{H}{\lambda}\right)^{2}+\frac{B}{B-s_{n}} \cdot \frac{H}{3}^{2}
\end{array}\right\}
$$




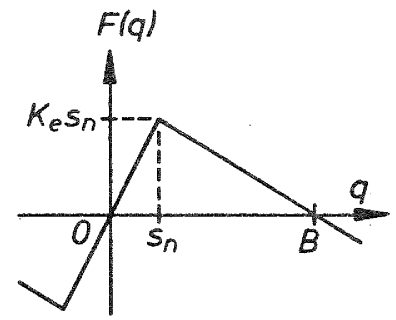

(a) Actual Porce

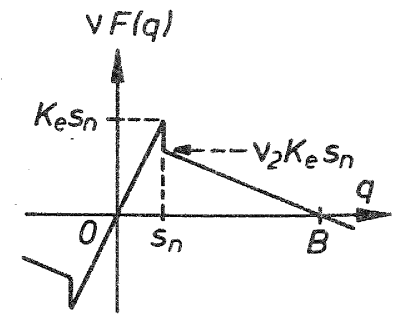

(b) Effective force

\section{Pigure 3: Base shear forces vs top displacement}

From $G_{B U}=G_{A U}$, we obtain

$\zeta_{U}=\frac{\int_{0}^{H} U \cdot y \cdot d y+\frac{B^{2}}{12}}{\int_{0}^{H} V \cdot y \cdot d y+\frac{B^{2}}{12}+\frac{B^{2}}{4} \cdot \frac{B}{B-s_{n}}}<1.0$

Similarly, the top velocity increases from $\dot{q}$ to $\dot{q} / \zeta$ at the instance of landing shown in Fig.2(a). Using a similar approach as above, $\zeta_{L}$
is pound to be

$\zeta_{L}=\frac{\int_{0}^{H} U \cdot y \cdot d y+\frac{B^{2}}{12}}{\int_{0}^{H} V \cdot y \cdot d y+\frac{B^{2}}{12}-\frac{B^{2}}{4} \cdot \frac{B}{B-s_{n}}}<1.0$

\subsection{Response to Sinusoidal Excitation}

To investigate the response predicted by the SDOF model, we consider a prismatic elastic body shown in Fig.4, where $\mathrm{h}$ means the damping factor before uplifting defined below.

$$
h=\frac{\gamma}{2 E} \cdot \sqrt{\frac{K_{e}}{M_{e}}}
$$

The fundamental period of the body before uplifting is $T=1.55 \mathrm{~s}$. Physical properties of the body are similar to those of stacked crates in Ref. (5).

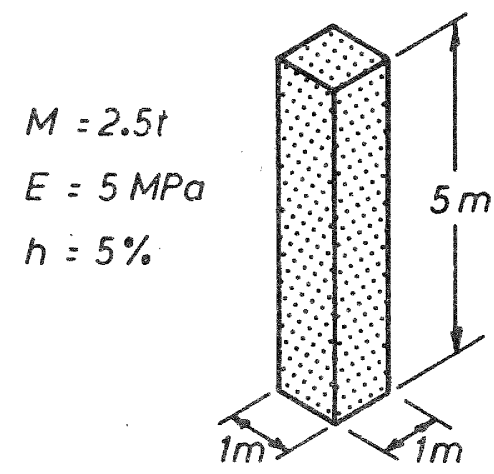

\section{Figure 4: Example Flexible rocking body}

The floor acceleration is sinusoidal as shown in Fig.5, with a period of $1.0 \mathrm{~s}$ and an amplitude of $5 \mathrm{~m} / \mathrm{s}^{2}$. The initial part of the floor acceleration was, however, replaced by third order function of time with duration of $0.412 \mathrm{~s}$ so that the velocity and the displacement of the floor would be also sinusoidal after $0.412 \mathrm{~s}$. (Note that imposing a sinusoidal acceleration with floor velocity and acceleration both zero at $t=0$ violates pure sinusoidal requirement.)

The equation of motion was solved step by step by a linear acceleration method $(B=1 / 6$ in Newmark's $B$ method), with a time interval $\Delta t=$ 0.02 s. Compensation for moment overshoot was made at every time step, where necessary.

The time history responses of the top displacement, its flexural and rocking components, and the top velocity are shown in Fig.6. These results will be compared with those of finite element analysis, later.

\section{EINITE ELEMENT ANALYSIS}

\subsection{Method of Analysis}

In order to verify the SDOF model, a finite element analysis was carried out on the elastic body identical to the previous section. The elastic body was partitioned into $2 \times 5$ elements as shown in Fig.7. Poisson's ratio was assumed as 0.25 , therefore the shear modulus was $\mathrm{G}=2 \mathrm{MPa}$. Prior to dynamic response analysis, the gravity force was statically imposed on every nodal point. follows:

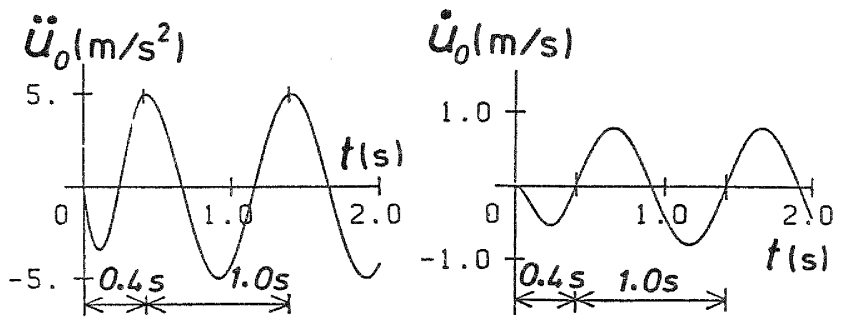

(a) Acceleration (b) Velocity

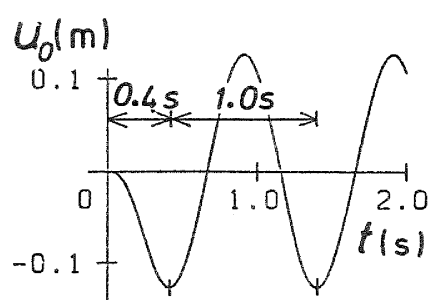

(c) Displacement 


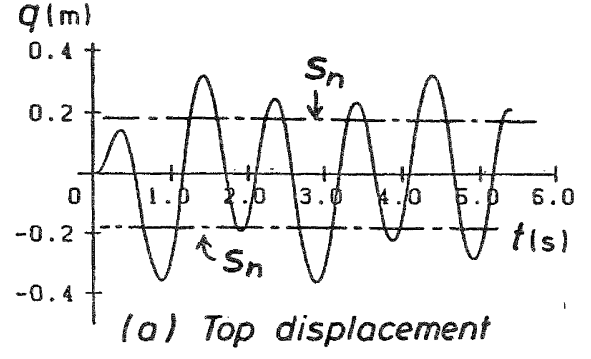

$s(m)$
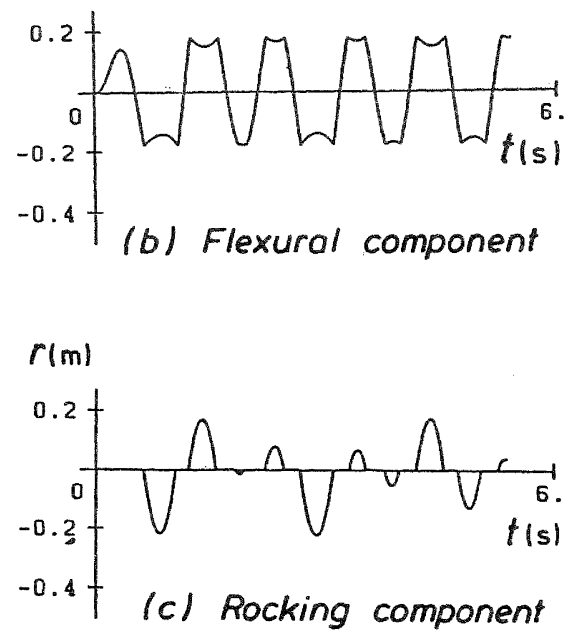

$q \dot{q}(\mathrm{~m} / \mathrm{s})$

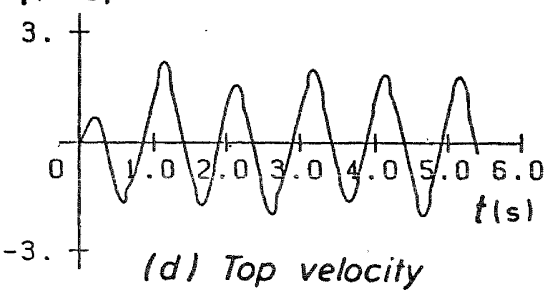

Figure 6: Response of SDOF model

$[M] u+[C] \dot{u}+[K] u=R-[M]\left(g+\ddot{u}_{0}\right)$

\section{where}

$[\mathrm{M}]=$ mass matrix

$[C]=$ damping matrix

$[\mathrm{K}]=$ stiffness matrix

$u=$ nodal displacement vector (vertical and horizontal)

$\mathrm{R}=$ floor reaction vector (at the base only)

$\mathrm{g}=$ gravity acceleration vector (vertical only)

$\ddot{u}_{0}=$ floor acceleration vector (horizontal only)

The damping matrix was assumed as stiffness proportional, i.e.

$$
[C]=\frac{h \cdot T}{\pi} \cdot[K]
$$

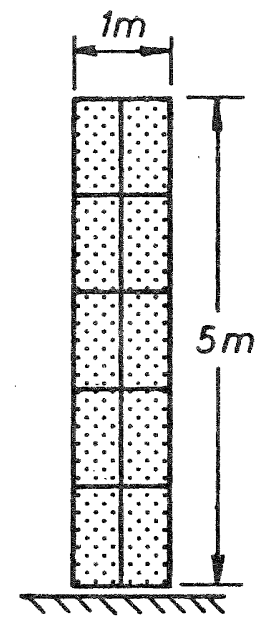

Figure 7: Finite element partition

where $h=0.05$ is the damping factor, and $T=$ $1.55 \mathrm{~s}$ is the flexural fundamental period before uplifting (these are the same as the previous section). The equation of motion was solved step by step by a linear acceleration method with a time interval $\Delta t=0.001 \mathrm{~s}$.

Every nodal point at the base of the body should be at one of the three states shown in Fig.8: free (= uplifting), slip (= move horizontally) or fixed (= stay at the position of the previous time step). The state was changed at the next time step in the following cases.

$$
\begin{array}{lll}
\text { Free } & \text {, and } u_{y} \leqq 0 & \rightarrow \text { Fix } \\
\text { Slip or Fix }, & \text { and } R_{y} \leqq 0 & \rightarrow \text { Free } \\
\text { Slip } & , R_{y}>0 \text { and } R_{x} \cdot{ }^{\circ} \geq 0 & \rightarrow \text { Fix } \\
\text { Fix } \quad R_{y}>0 \text { and } R_{x}>\mu_{s} \cdot R_{y} & >\text { slip } \\
\quad\left(\mu_{s}=\text { static coefficient of friction }\right)
\end{array}
$$

At the instance of Free $\rightarrow \mathrm{Fix}$, the vertical displacement $u_{u}$ was backed to zero. Static and kinetic friction coefficients, $\mu_{s}$ and $\mu_{k}$ were assumed as 0.8 and 0.4 , respectivelys.

\subsection{Results}

Deformed shapes of the elastic body from $t=0.9 \mathrm{~s}$ to $t=1.4 \mathrm{~s}$ are exaggerated by five times and shown in Fig.9 with time interval of
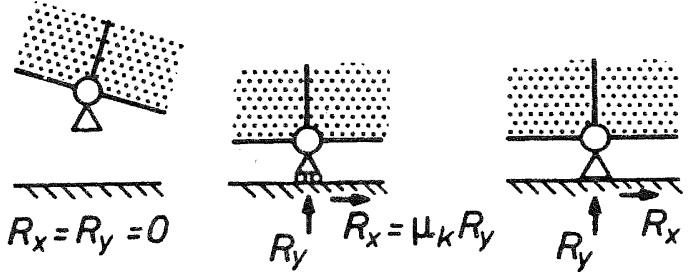

(a) Free (b) Slip

(c) Fixed

Figure 8: Three states of nodal point at base 


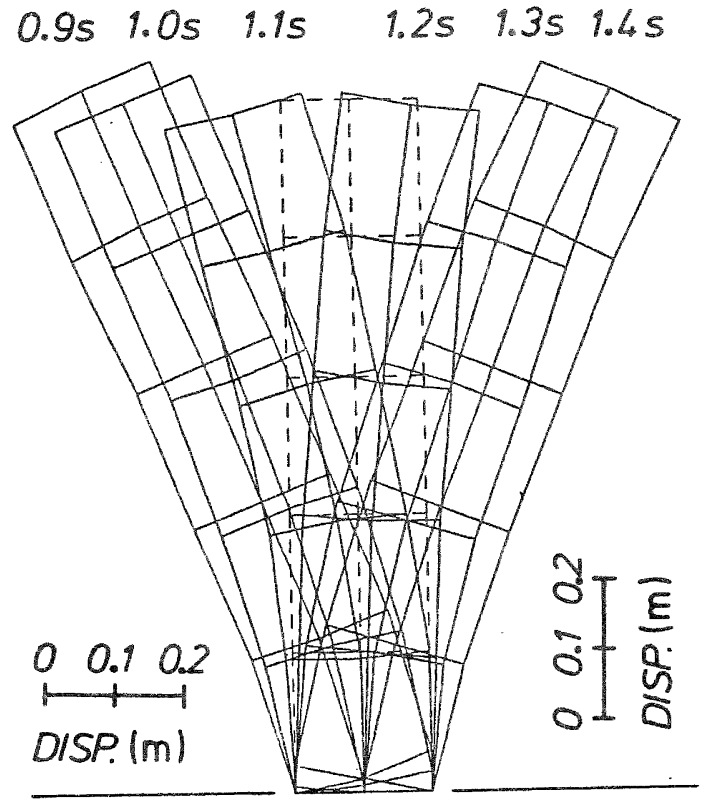

Figure 9: Deformed shape of body

(x 5)

$0.1 \mathrm{~s}$. At $t=0.9 \mathrm{~s}$ and $1.0 \mathrm{~s}$, the body is rocking around the left edge. 'The body is more straight (or its flexural deformation is smaller) at this time than at $t=1.1 \mathrm{~s}$ when the base rests on the floor.

The time history of top displacement relative to the floor is shown in Fig.10. It is decomposed into flexural, rocking, slipping and shearing components and their time history is shown in Fig.11. The chained lines in Fig.10 and Fig.11(a) represent the theoretical uplifting displacement \pm $s_{n}$. The flexural component does not go far beyond $\pm s$ and it decreases as the rocking component increases, making a dent in the response wave as was observed in the SDOF results. The slipping and shearing components of the FEM results are negligibly small.

The relationship between the top displacement $q$ and the rocking component $r$ is shown in Fig.12. The rocking component tends to exist as long as the top displacement is iarger than $s_{n}$, as it does in the SDOF model.

The relationship between the flexural component $s$ and the rocking component $r$ is shown in Fig.13. The flexural component tends to decrease as the rocking component increases, as 1 t does in the SDOF model.

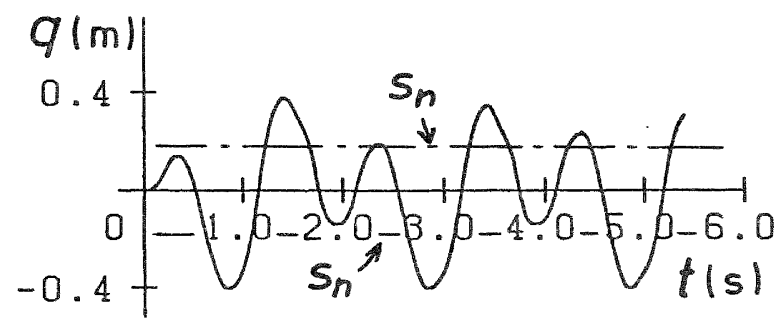

Figure 10: Top displacement response (FEM)

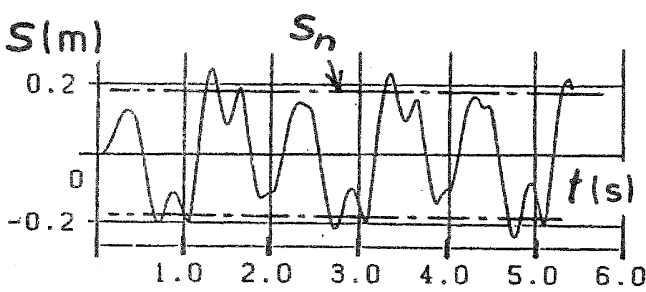

(a) Flexural component

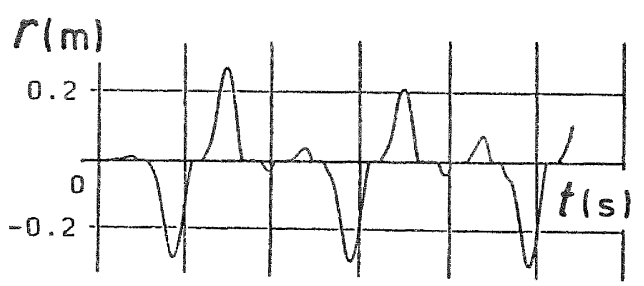

(b) Rocking component
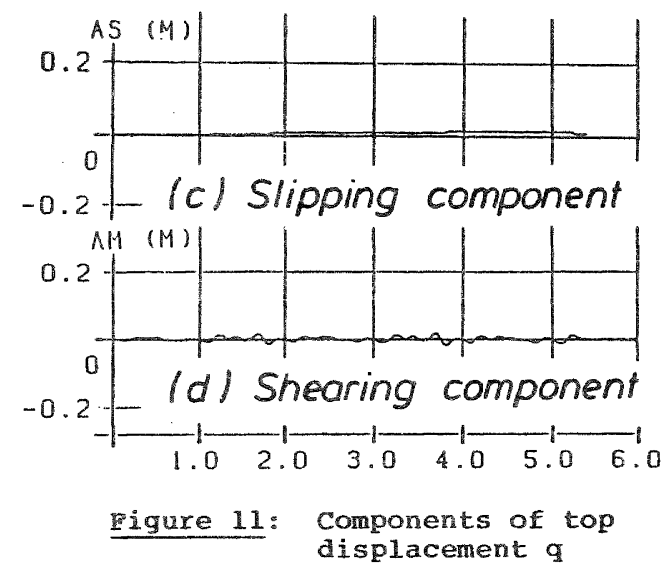

The time history of top velocity $q$ is shown in Fig.14. Those of angular momentum around left and right edges, $G_{L}$ and $G_{R}$, are shown in Fig.15. The side of $G_{L}<O^{R}$ (Lower half) of Fig:15(a) includes all the instances of landings at the left edge and upliftings around the left edge. Similarly, the $G_{R}>0$ side (upper half) of Fig.15(b) includes all the instances of landings at and "upliftings around the right edge. The wave shape of both halves looks smooth enough; while that of top velocity has several peaks and steps. Therefore, we can judge that the angular momentum is conserved at the instances of uplifting and landing in accordance with Assumption 4.

\subsection{Discussion}

Besides the similarities stated in the previous section, we should also note some disagreements between the FEM results and the SDOF model's results as follows.

(a) In Figs.11 and 13, the plexural component tends to go beyond $s_{n}$ when the rocking motion starts to appear.

(b) As the rocking component increases in Fig.13, the flexural component tends to decrease at a larger rate than the SDOF model predicts. (This is equivalent to the dents in the response wave of the flexural component in Fig.11(a) being deeper than those of the SDOF model:) 


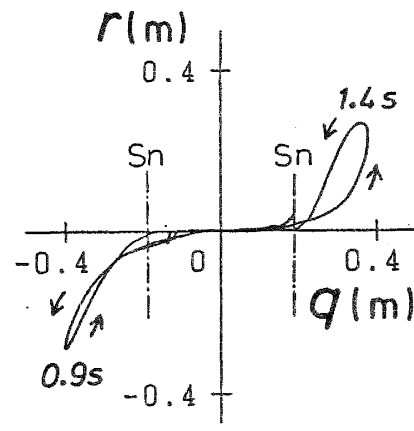

(a) $t=0$ to $2.7 \mathrm{~s}$

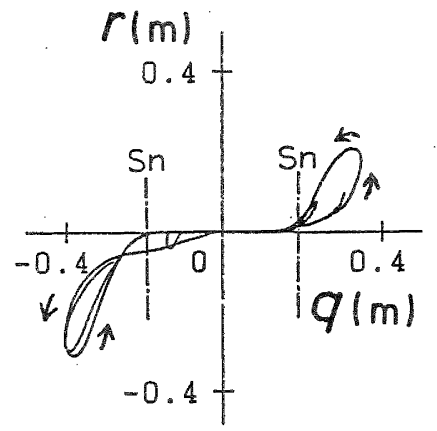

(b) $t=2.7 \mathrm{~s}$ to $5.4 \mathrm{~s}$

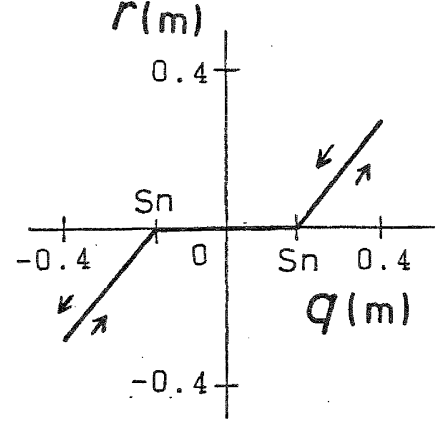

(c) SDOF model

Figure 12: Top displacement $q$ vs rocking component $r$

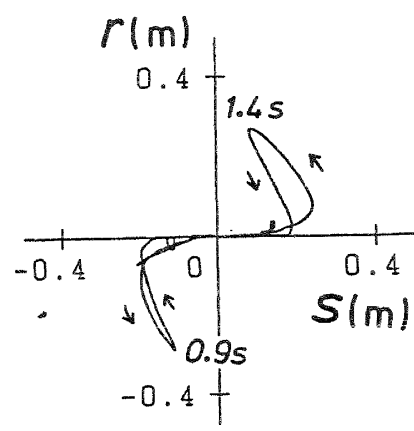

(a) $t=0$ to $2.7 \mathrm{~s}$

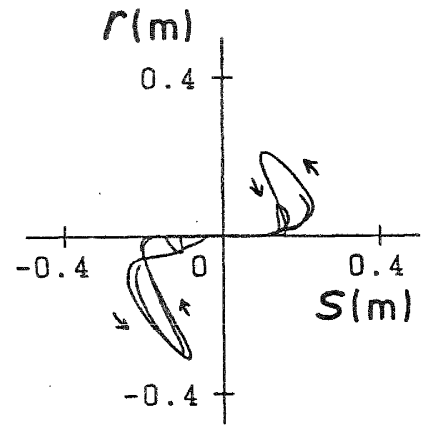

(b) $t=2.7 \mathrm{~s}$ to $5.4 \mathrm{~s}$

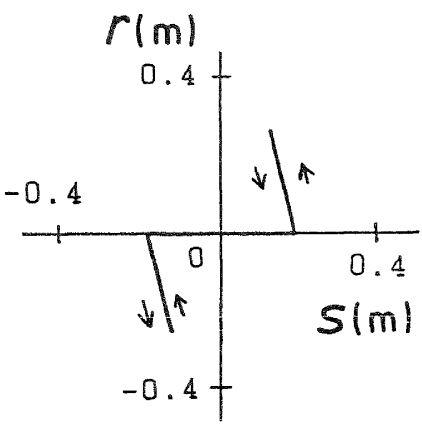

(c) SDOF model

Figure 13: Flexural component s vs rocking component $r$

(c) In Fig.14, the top velocity response has sharper peaks than in the SDOF response.

The disagreement (a) should be partly attributable to the fact that the vibration phase of top movement was just opposite to that of floor movement. Therefore, distribution of overturning force $w$ in Eq.(6) should have been considered as Fig.16(a) rather than that shown in Fig.16(b). Apparently, the overturning force of Fig:16(a) yields a larger deflection than that of Fig.16(b) when it yields the same amount of overturning moment. This effect would be the contrary if the period of floor motion were shorter than the fundamental period of the body as shown in Fig.16(c). This effect would be smaller if the period of floor motion were nearer to that of the body because the dynamic magnification factor would be larger.

The disagreement (b) and part of (a) should be attributable to the change of velocity distribution at the instance of uplifting in the SDOF model as shown in Fig.17. The difference of velocity distribution shown in Fig.17(c) should cause a higher vibration mode which increases the flexural deformation at the beginning of uplifting and decreases it as the rocking component increases.
The disagreement (c) should again be attributable to the change of velocity distribution, but this time, at the instance of landing. It should induce a higher mode, which would cause sharper peaks in the top velocity response.

Consideration of these effects, however, requires a much more complicated model than that presented in this paper. On the basis of the comparison between the SDOF and the FEM results shown here, the author does not feel that these shortcomings are particularly significant.
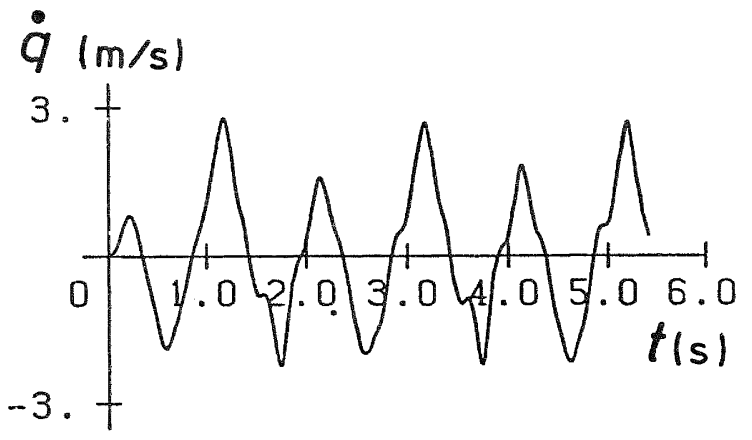

Figure 14: Top velocity response 

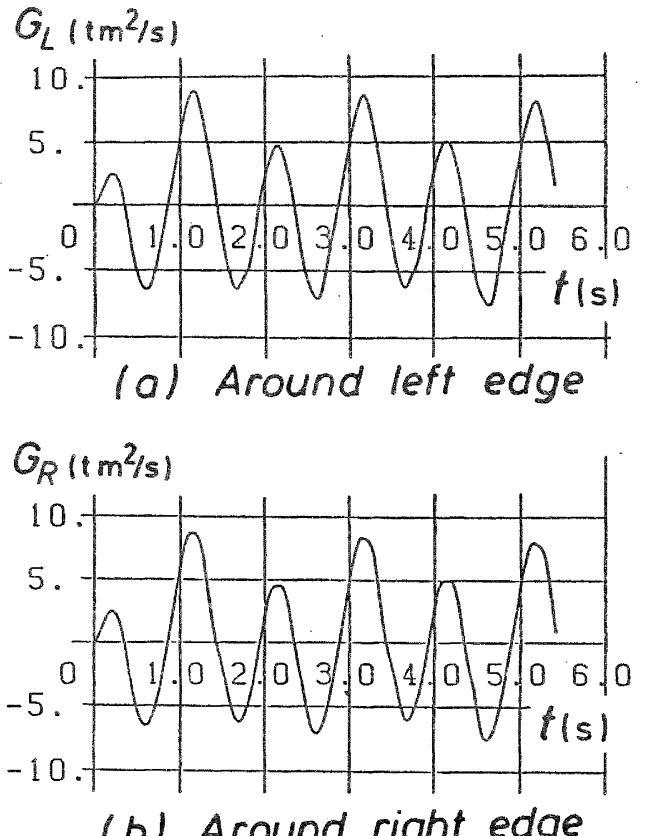

(b) Around right edge

\section{Figure 15: Angular momentum response}

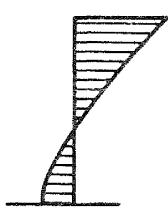

(a) With floor acceleration
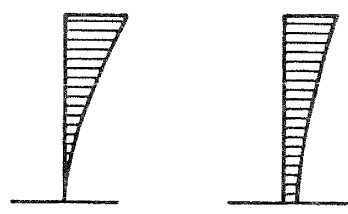

(b) Proportional (c) With to U(y)

\section{Pigure 16: Distribution of overturning force}
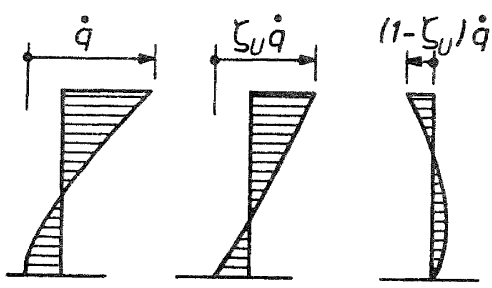

\section{(a) Before upliffing}

(b) After uplifting

Figure 17:

\section{FUTURE RESEARCH NEEDS}

The presented SDOF model should be utilised for parametric studies to establish the criteria to prevent overturning of elastic bodies by earthquake excitations. The criteria should have continuity to those for rigid bodies such as Ishiyama's (6).

The presented SDOF model for a single elastic column, however, should be greatly modified when we investigate the seismic behaviour of an assemblage consisting of a number of adjacent elastic columns, such as a piled stack of crates which suffered damage during the 1978 Miyagi-ken-oki earthquake ( $i$ ). In such a case; we cannot ignore the effects of collision and friction between the neighbouring columns (5). Modelling of these effects is also recommended.

\section{CONCLUSIONS}

(1) Rocking motion of slender elastic body on a rigid floor can be described by a single degree of freedom model, which assumes the vibration mode to be a linear combination of flexural and rocking modes. The model provides a simple means for assessing the seismic response of elastic rocking bodies, and would be particularly suitable for parametric studies.

(2) Rocking motion occurs when the top displacement relative to the floor exceeds a certain limit, which can be calculated from the physical properties of the body.

(3) Flexural deformation decreases as rocking displacement increases, which relation can also be calculated from the physical properties of the body.

(4) Angular momentum is conserved at the instances of uplifting and landing.

\section{ACKNOWLEDGEMENTS}

The author very hizhly appreciates the contributions by Dr M.J.N. Priestiey of the University of Canterbury for critical reading of the manuscript and a great number of suggestions to make this paper readable. Encouragement by Prof. $R$. Park and Prof. T. Paulay is also acknowledged. Technical suggestions by Pror. K. Takiguchi of Tokyo Institute of Technology are highly appreciated. Finally, the author expresses his gratitute to Mrs V.J. Grey for draughting the figures and to Miss C.M. Reid for typing the manuscript.

\section{REFERENCES}

(1) Architectural Institute of Japan, Report on the Damage by 1978 Miyagi-ken-oki Earthquake (in Japanese), Tokyo, Japan, $1980,0.838$

(2) Aslam, M., Godden, W.G. and Scalise, D.T. "Earthquake Rocking Response of Rigid Bodies", Journal of the Structural Division, ASCE, Vol. 106, No. ST2, February 1980 , pp. 377-392.

(3) Clough, R. and Penzien, J., Dynamics of Structures, McGraw-Hill Inc., New York, N.Y., 1975, pp. 301-314.

(4) Housner, G.W., "The Behavior of Inverted Pendulum Structures during Earthquakes", Bulletin of the Seismological Society of America, Vol. 53, No. 2, February 1963, pp. $403-417$. 
26

(5) Ichinose, T., Murakami, M., Ohami, K. and Hosokawa, Y., "Behavior of Stacked Crates during Earthquake", Proceedings of 8th World Conference $\frac{\text { on }}{n}$ Earthquake $1229-1236$.

(6) Ishiyama, Y., Motion of Rigid Bodies and Criteria for Overturning by Earthquake Excitations", Bulletin of the New Zealand National Society for Earthquake Engineering, Vol. 17, No. 1, March 1984, pp. 24-37.

APPENDIX

Reference to Classical Model for Rigid Bodies

The presented SDOF model converges to the classical model for slender rigid bodies when the Young' $S$ modulus $E$ becomes infinitely large.

[Equation of motion] follows.

From Eq. (8), $s_{n}$ and EI. $s_{n}$ converge as

$$
s_{n}=0
$$

$$
E I \cdot S_{n}=\left(\frac{H}{\lambda}\right)^{2} \cdot \frac{M g B}{2}
$$

Therefore, $F(q)$ of Eq. (17b) converges to

$F(q)=\frac{M g B}{2} \cdot\left( \pm 1-\frac{q}{B}\right):\left(\frac{\lambda}{H}\right)^{2} \cdot \int_{0}^{H} U \cdot d y$

From Eqs.(11b) and (13),

$$
v_{2}=\frac{3}{\lambda^{2}} \text { and } \beta_{2}=\frac{3}{2}
$$

Consequently, the equation of motion Eq.(16) reduces to

$$
M \cdot \ddot{q}+\left( \pm 1-\frac{q}{B}\right) \frac{3 M g B}{2 H}=-\frac{3}{2} M \cdot \ddot{u}_{0}
$$

The exact equation of motion for the rigid body shown in Fig.A1 is as follows.

$$
\begin{gathered}
M: \sqrt{H^{2}+B^{2}} \cdot \ddot{\theta}+\frac{3}{2} M g \cdot \sin ( \pm \alpha-\theta) \\
=-\frac{3}{2} M \ddot{u}_{0} \cdot \cos ( \pm \alpha-\theta)
\end{gathered}
$$

If the body is slender enough to be $(B / H)^{2}=0$ and $(q / H)^{2} \approx 0$, we obtain sin $( \pm \alpha-\theta)$ $\approx \pm 1-Q / B$ and $\cos ( \pm \alpha-\theta)=1$, and Eq. (A6) converges to Eq. (A5).

In order that the SDOF model may converge to the exact equation of motion for rigid body, the effects of vertical displacement should also be included in Assumption 1 of section 2.1 .

[Conservation of angular momentum]

From Eqs.(21), (22) and (20), the ratio $\zeta_{\mathrm{U}} / \zeta_{\mathrm{L}}$ converges to the following value.

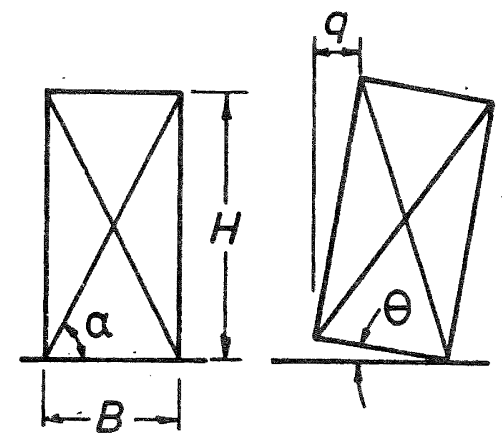

Figure Al: Uplifting of rigid body

$$
\frac{\zeta_{U}}{\zeta_{L}}=\frac{2 H^{2}-B^{2}}{2\left(H^{2}+B^{2}\right)}
$$

This is exactly the same as the ratio of velocity decrease of a rigid body at the instance of landing.

\section{LIST OF SYMBOLS}

E

$=$ Young's modulus

$F(q) \quad=$ base shear force

$G_{A U}, G_{B U}=$ angular momentum after or before uplifting

$G_{L}, G_{R}=$ angular momentum around left or right toe

= gravity acceleration

$\begin{array}{ll}8 & =\text { gravity accelera } \\ \mathrm{H} & =\text { height of body }\end{array}$

h $=$ damping factor for pre-rocking vibration

I $=$ moment of inertia of section

$\mathrm{K}_{\mathrm{e}} \quad=$ base shear stiffness

$\mathrm{L}_{\mathrm{C}}, \mathrm{L}_{\mathrm{S}} \quad=$ constants $\left(L_{c}=0.3671\right.$ and $\left.L_{s}=1 / 2\right)$

M = total mass of body

$\mathrm{M}_{\mathrm{e}} \quad=$ effective mass of body

$m_{B} \quad=$ elastic bending moment at the bottom of body

$\mathrm{m}_{\mathrm{R}} \quad$ = resisting moment by gravity force

$\mathrm{m}_{\mathrm{T}} \quad=$ overturning moment by inertia plus damping force

$q(t)=$ horizontal displacement at the top of body relative to floor

$r(t), s(t)=$ rocking and flexural components of tip deflection $q(t)$

$s_{n} \quad=$ tip deflection at instant of uplift 


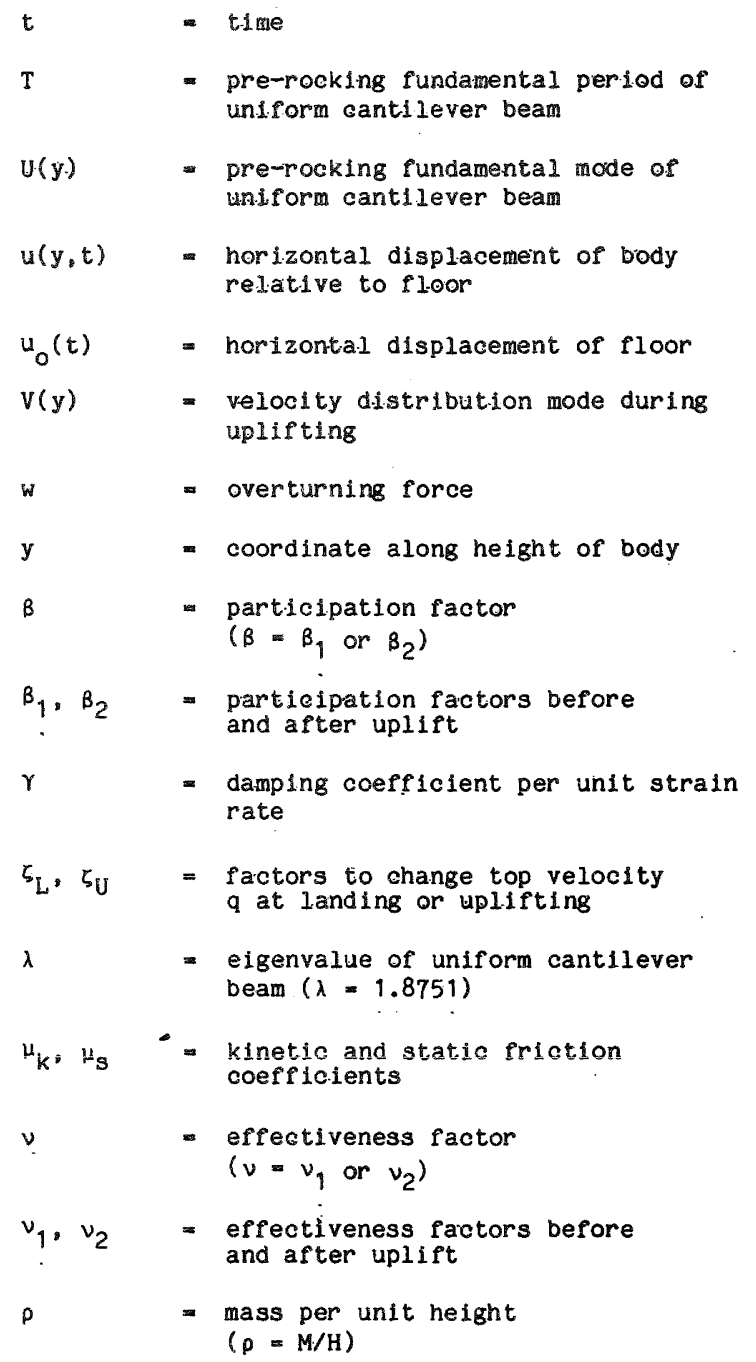

